Clinical Case Report

\title{
Osteoid Osteoma of the Metacarpal - Difficulties in the Diagnosis: A Case Report
}

\author{
Arnela Hairlahović ${ }^{1}$, Sabaheta Jonuzović-Prošićn ${ }^{2}$ Jasmina Osmančević-Muminović ${ }^{1}$, Salih Mesić ${ }^{3}$
}

${ }^{1}$ Department of Paediatrics, Community

Health Centre Cazin, Bosnia and

Herzegovina, ${ }^{2}$ Department of Family

Medicine, Community Health Centre

Cazin, Bosnia and Herzegovina,

${ }^{3}$ Department of Radiology, Community

Health Centre Cazin, Bosnia and

Herzegovina

Correspondence:

arnela_hair@outlook.com

Tel: + 38761436678

Fax.: +38737539027

Received: July 25, 2019

Accepted: September 5, 2019

Key Words: Osteoid Osteoma - Metacarpal - Wrist Pain.
Objective - To present a rare case of osteoid osteoma of the metacarpal bone and to emphasize the importance of correlation of radiological analysis with anamnestic data and clinical findings. Case Report - We reported the case of osteoid osteoma of the metacarpal bone in a 14-year old girl. The patient, a volleyball player, presented with pain in the left hand. At first, nonsteroidal anti-inflammatory drugs relieved the pain but over the time it became more intense and was accompanied with swelling. On the basis of magnetic resonance imaging (MRI), the case was misdiagnosed as a status post-fracture of three metacarpal bones. After inappropriate treatment with physical therapy, the patient's condition worsened, thus multiple laboratory tests and radiology imaging techniques were performed to achieve the right diagnosis. Diagnosis of osteoid osteoma was finally made by CTscan and appropriate treatment by surgical ablation was performed. Conclusion - Osteoid osteoma rarely occurs in metacarpal bones. Physicians should be aware of the atypical clinical presentation of this benign lesion in the metacarpal bones.

\section{Introduction}

Osteoid osteoma is a benign osteoblastic bone tumor typically occurring in younger patients in their second or third decade of life. It predominantly affects males, though patients of all ages and genders can be affected (1). It was first described by Jaffe in 1935 and it is the third most common benign lesion of bone (2). Osteoid osteoma is a small spherical tumor with a diameter of $1.5 \mathrm{~cm}$ or less (3). It is typically a cortical lesion, located in the diaphysis or metaphysis of long bones, the femoral neck is the most common location. However it may be subperiosteal, endosteal or medullary, with most authors considering these locations to result from migration from the typical cortical origin (4).
Five to $15 \%$ of osteoid osteoma occur in the hand and wrist, most commonly in the proximal phalanx and carpus(5). It is reported that the metacarpals are the least common site for this tumor (6).

Typical radiographic findings of osteoid osteoma include an intracortical nidus, which may display a variable amount of mineralization, accompanied by cortical thickening and reactive sclerosis in a long bone shaft. Carpal and tarsal osteoid osteoma may display less reactive sclerosis, a finding that can be misleading (7).

Prostaglandins are found in the nidus at levels 100 to 1000 times that of normal tissue. They induce vasodilation and resultant increased capillary permeability in the tissues surrounding the lesion and are believed to 
mediate tumor-related pain, classically described as night pains relieved by salicylates (3). The patients most often complaints of pain which is nocturnal, intermittent and typically relieved by taking aspirin (6). The clinical diagnosis of osteoid osteoma in metacarpals is often missed or delayed. This is due to the fact that the clinical, radiological and histological features of osteoid osteoma in metacarpal bones may differ from that in long bones (8).

Here we present a rare case of osteoid osteoma of the metacarpal bone in a young patient.

\section{Case Report}

A 14-year-old right-handed girl presented with swelling and pain in her left hand. The pain had started one and a half years before when she came to the doctor for the first time. In view of the fact that the patient was attending regular volleyball practice and suspecting an injury, an X-ray of the hand was done. However, the X-ray came back clean without evidence of fracture, so nonsteroidal anti-inflammatory drug (NSAID) - ibuprofen, was prescribed for pain relief. Since the pain has decreased no further diagnostics were ordered. Three months back, occasional pain became constant, more intense and accompanied by swelling of the hand. Ibuprofen, which had been used daily (600-1200 $\mathrm{mg}$ ) for longer than one month, brought no longer relief. The pain radiated to the left shoulder and the back, worsened with activity and for that reason, the patient stopped with the volleyball practice. The patient denied earlier injuries, morning stiffness or pain in other joints.

Physical examination revealed swelling on dorsum of the left hand with tenderness as well as spreading pain along the arm when palpated. Left hand grip was weaker than the right hand one. The musculature of the left hand was hypotrophic in comparison with the right hand and the left elbow circumference was $1.5 \mathrm{~cm}$ less than the circumference of the right elbow. The left scapula was positioned higher relative to the right, and painful in all the points, as established by palpation.

All of the ordered laboratory tests came back clean (full blood work, erythrocyte sedimentation rate, and rheumatoid factor (RF)), with the exception of antistreptolysin $\mathrm{O}$ titer (ASTO) which was positive at $400 \mathrm{IU} / \mathrm{mL}$. Comparative X-ray of both hands eliminated a suspected fracture and due to thickening of corticalis II, III and IV metacarpal bones of the left hand the radiologist suggested further diagnostic tests and imaging (Fig. 1.)

The first examination by orthopedist did not prompt indication for further orthopedic treatment, while the physiatrist wanted to examine the possibility that a ganglion cyst has formed. Additional diagnostics were conducted: test for brucellosis came back negative, nasal and throat swab tests were clean. Due to an increased ASTO value, heart ultrasound was also done, showing normal condition. The ultrasound of the soft structures on the left hand showed signs of cutis and subcutis lymphostasis, as well as signs of peritendinitis. Furthermore, the MR scan of the left hand was taken (one month after the $\mathrm{x}$ ray), showing the pathological signals of the diaphysis on II, III and IV metacarpal bones, with thickened corticalis, suggesting the diagnosis of a post-fracture condition. Based on these findings, the patient was sent for five physical therapy treatments and cryotherapy was also advised (Fig. 2).

Following the physical therapy treatment, the pain in the hand and shoulder worsened and could no longer be relieved by oral analgesic therapy, so the patient was sent to the Pediatric Clinic of the Clinical Centre for further examination and treatment. Due to suspected rheumatoid etiology of the pain and swelling, additional laboratory tests were 


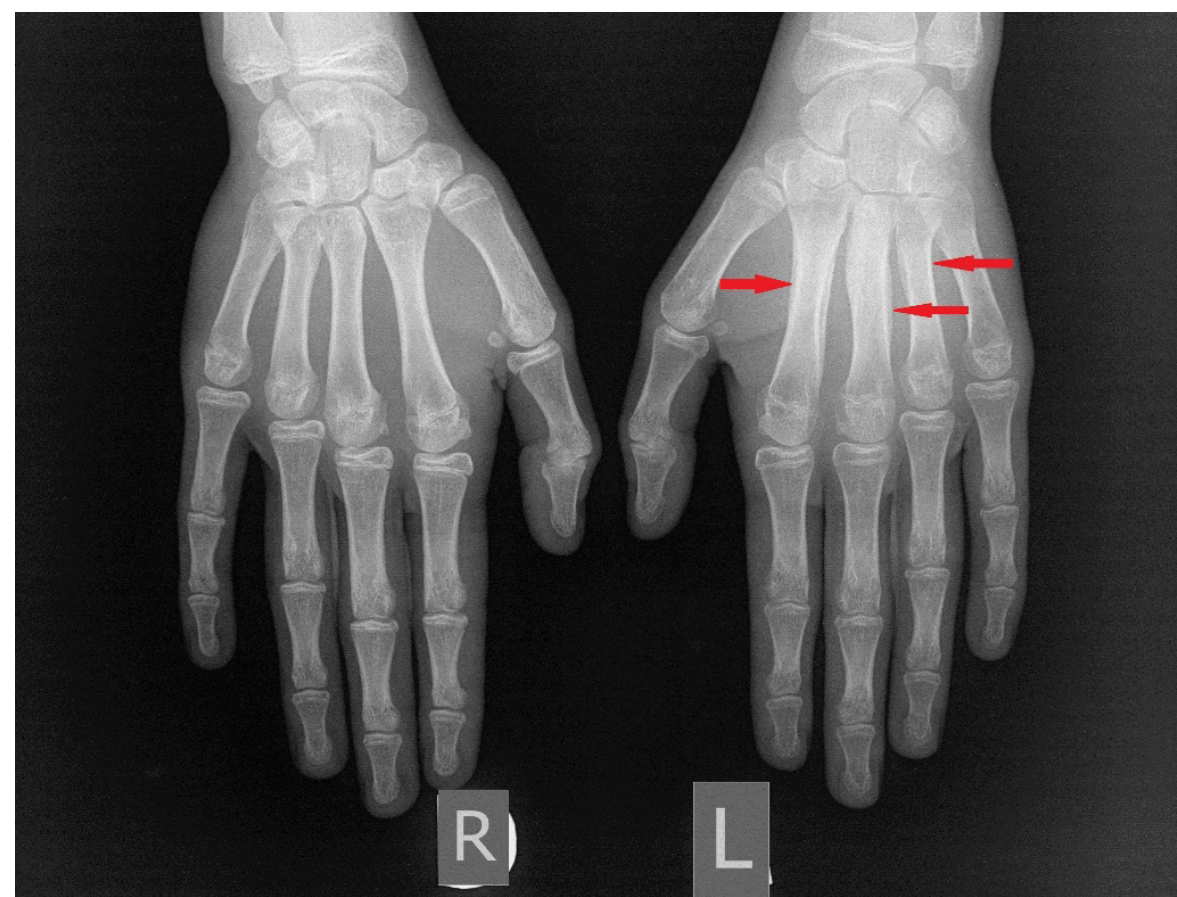

Fig. 1. Plain Radiographs of Both Hands Showing Cortical Thickening of II, III and IV Metacarpal Bones. Sclerotic Increas Area at the Base of III Metacarpal Bone Covers Possible Nidus.

done. The following tests were performed: peripheral blood smear, ANA IFT-IFT Hep2 cells, HLA-B27, 24-h proteinuria, eGFR, proteinogram, Serum protein electrophoresis, rheumatism tests, total IgE, Chlamydia pneumoniae serology, Mycoplasma pneumoniae serology, CMV and EBV serology, urinoculture, coproculture, stool benzidine, nasal and throat swab, PTH, osteocalcin and beta cross laps. All laboratory tests came back clean, with the exception of positive IgG antibodies to CMV (IgM was negative) and the beta cross laps which had higher than normal value $(1.37 \mathrm{ug} / \mathrm{L}$, whereas normal value is $<0.57 \mathrm{ug} / \mathrm{L})$.

The ultrasound of the ankle joints, feet, elbows, both shoulders, and abdomen was performed but no argument was found in favor of a rheumatic disease. Furthermore, the ultrasound of peripheral lymph nodes pointed out reactively changed lymph nodes on

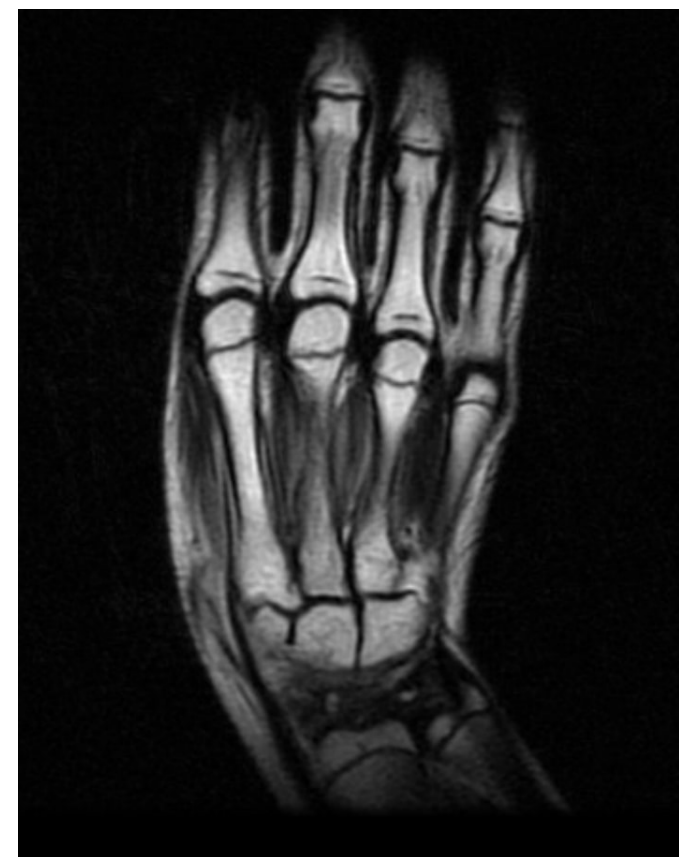

Fig. 2. Coronal MRI Scan Reveals Bone Oedema and Cortical Thickening of the Diaphysis of II, III and IV Metacarpal Bone, with no Pathologic Finding in Cortex and Medulla Such as Nidus. 
the neck, and lymph nodes with chronically changed echo characteristics bilaterally in axillary and inguinal regions. In view of the unclear clinical picture and the degree of difficulties which were not in correlation with the radiological examination (post-fracture condition of II, III and IV metacarpal bones), the orthopedist requested a joint examination with a rheumatologist and a radiologist, and consultations with a hemato-oncologist. At this point, no specific diagnosis could be established.

Looking at the history of the disease and previously conducted tests and radiological examination results, the hemato-oncologist did not identify elements of hemato-oncological disease, while the pediatrician endocrinologist recommended making a new X- ray of the wrist and hand. The X-ray revealed normal bone maturation, and noted a round, more dense zone at the base of III metacarpal bone of the left hand, with open etiology (Fig. 3). Due to hypotrophy of the entire left arm musculature, a mild deficit of the full extension of the left elbow and discrete weakness in the gross motor strength of the left hand, the physiatrist suggested doing EMG of the left hand and left arm, as well as X-ray of the neck spine in two directions. The X-ray of the neck spine came back clean and EMG of the upper left extremity was supporting axonal lesion of the ulnar nerve with a minimal bloc of medianus linking paracubitally. Skeletal scintigraphy was also performed, showing a focus in the area of the left metacarpal bones suggesting an acute process, possible inflam-

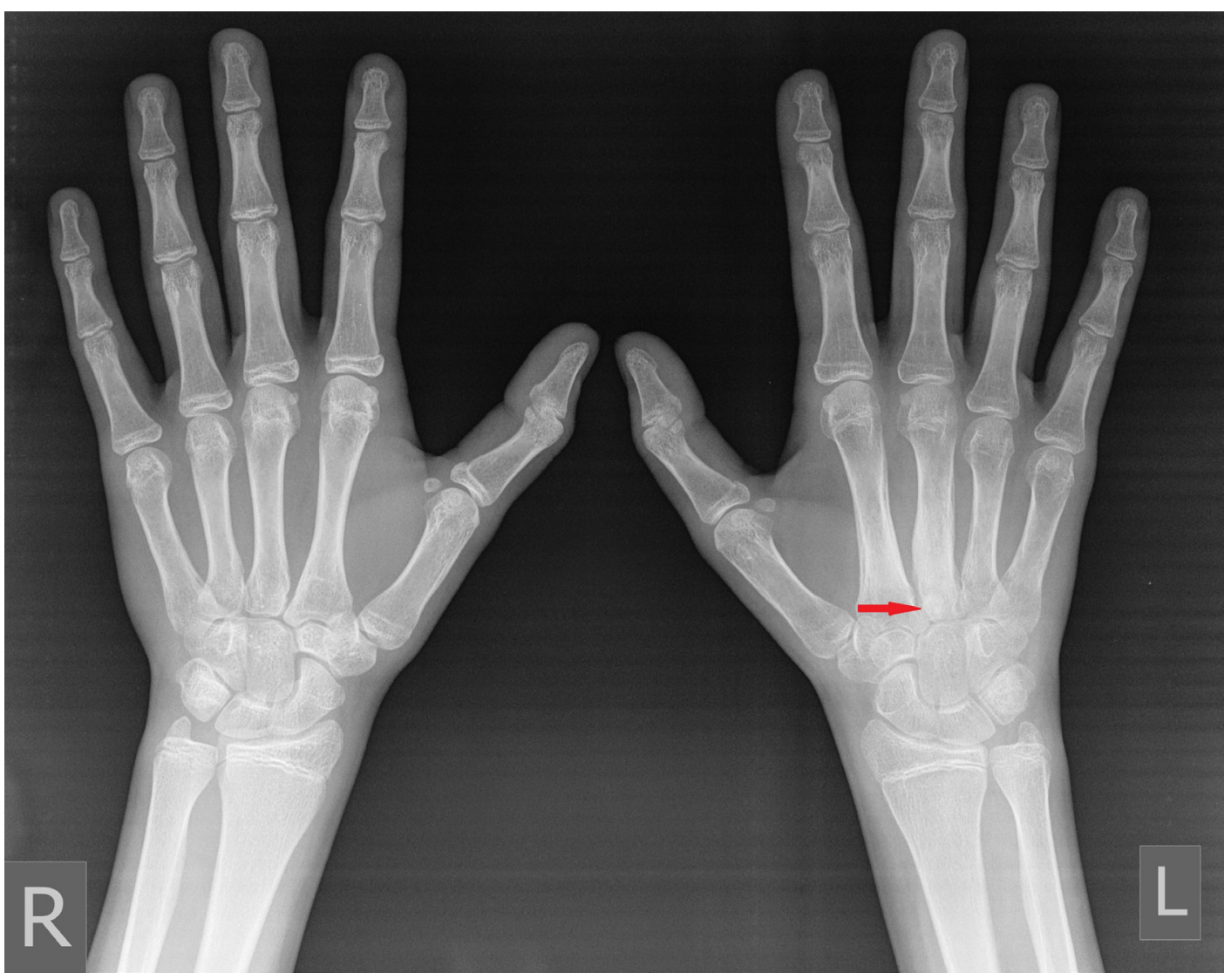

Fig. 3. Plain Radiograps of Both Hands Showing Round, More Dense Zone at the Base of III Metacarpal Bone of the Left Hand. 


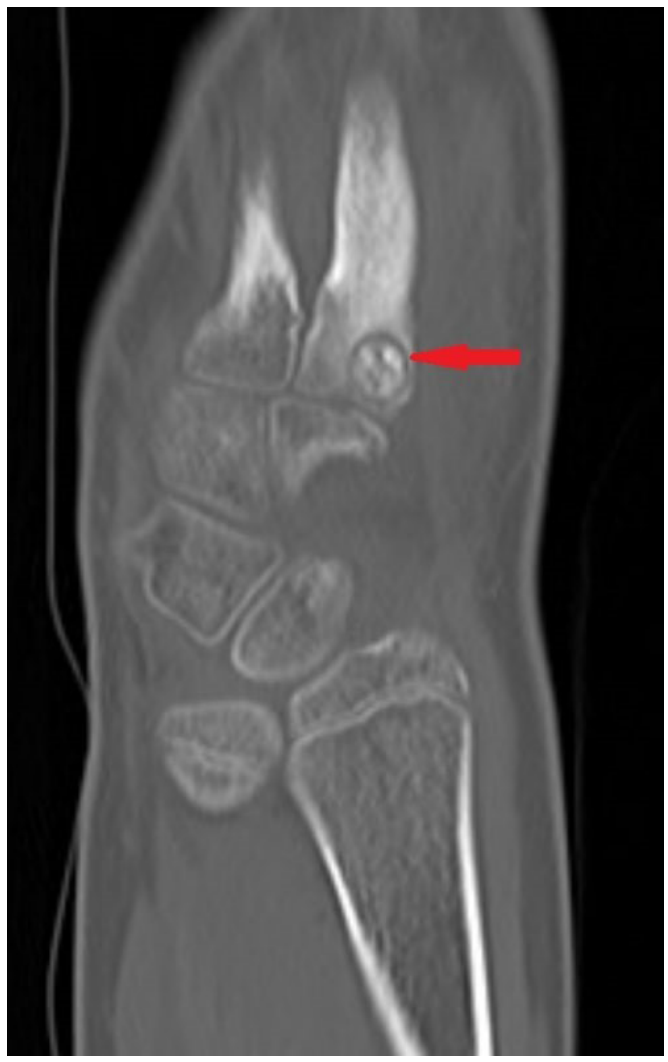

Fig. 4. CT of the Left Hand Showing the Well-Defined, Ring-Like Nidus on the Basis of the III Metacarpal Bone, which Is a Typical Presentation of the Osteoid Osteoma.

mation. In addition, moderate accumulation focus was found in the left shoulder.

Subsequently, a joint consultative meeting of rheumatologists, orthopedists, children's radiologist, and hemato-oncologist was held. It was concluded that additionally an MRI exam of the shoulder girdle and left hand CT should be done. The MRI of the left shoulder did not indicate any significant pathomorphological changes on the bones or muscles of the shoulder girdle, while axillary lymphadenopathy was described as reactive. The CT of the left hand showed a zone of changed density in the area of the base of III metacarpal bone which, according to CT characteristics, primarily corresponded to an osteoid osteoma, as well as visible deformity of III metacarpal bone with changes to II, III and IV metacarpal bones with changed corticalis (Fig. 4).

As the CT exam indicated changes in line with osteoid osteoma type, the patient was transferred to the Orthopedic Clinic to undergo a surgery. After extirpation, the tumor bone sample was sent for pathohistological analysis which confirmed the diagnosis. The patient's pain resolved in the first $24 \mathrm{~h}$ after surgery and she no longer needed analgesics, while the scope of movement in the hand recovered. Therefore, the correct diagnosis was made about five months after the first examination of the patient. A control X-ray was made three months after the surgery, showing the signs of bone overgrowth in the place of the previous tumor growth, and the patient has had no further difficulties.

\section{Discussion}

Osteoid osteoma accounts for around 5\% of all bone tumors and $11 \%$ of benign bone tumors (9). Between $19 \%$ and $31 \%$ of all osteoid osteoma occur in the upper extremity while lesions in the hand and wrist comprise $5 \%$ to $15 \%$ (10). All types of osteoid osteoma (cortical, medullary or subperiosteal) may occur in the metacarpal, metatarsal, and phalangeal bones. Because of the proximity of the bones in the hands and feet, an inflammatory reaction that originates from one lesion often spreads to adjacent bones and joints. Moreover, soft tissue swelling may be prominent which may resemble infection or inflammatory arthritis (7).

The patients usually present with pain and swelling (6). If osteoid osteoma involves a bone in a subcutaneous location, erythema and tenderness are also present. In the hand and wrist, point tenderness is characteristic (10). In most cases, pain is described as a dull and unremitting ache that starts off as mild and intermittent that gradually increases in 
intensity and persistence. The disparity between intense pain and the small physical size of the lesion is probably explained by the presence of nerve fibers and high vascularity within the nidal and perinidal tissues, which is a unique feature in this tumor (3).

Osteoid osteoma that occurs within or near a joint is rare. Due to the rarity and nonspecificity of its clinical presentation such as pain, joint tenderness, joint effusion even monoarthritis, diagnosis may be difficult and mistaken for other etiologies such as inflammatory or infectious arthritis, aseptic osteonecrosis, fatigue fracture or radicular syndrome (11). If lesion continues to progress, the patient can present with flexion contracture and a decreased range of motion (9). The referred pain, simultaneous muscle atrophy and decreased deep tendon reflexes in the affected limb may erroneously suggest the presence of a primary neurological disorder $(9,12)$.

In the here presented case, the unclear clinical presentation alluded to a rheumatoid etiology but performed tests were negative. EMG performed due to suspicion of neurological disease has shown axonal lesion of the ulnar nerve caused by compression of osteoma on the deep nerve branches.

Typical findings of osteoid osteoma are an intracortical nidus with fusiform cortical thickening, reactive sclerosis, and bone marrow edema (7). However, osteoid osteoma may have an imaging appearance similar to those of other conditions, which can lead to confusion. Subperiosteal osteoid osteomas can produce an extensive aggressive periosteal reaction, whereas intraarticular lesions typically cause relatively little periosteal new bone formation (13). In the metacarpals and phalanges, some lesions may display no lytic nidus on any imaging modality and there may be an absence of reactive bone sclerosis (8).

Due to the unusual radiographic presentation of some osteoid osteoma, many are not diagnosed by plain radiography. It is reported that between $24 \%$ and $29 \%$ ofosteoid osteomas are not demonstrable on plain radiographs $(10,14)$. In such cases, CT, bone scintigraphy and magnetic resonance imaging are useful in making the correct diagnosis. However, precise localization of the osteoma on MRI may not be easily detectable in 35\% of the cases, as it is often hidden by the associated perilesional edema, while in 50\% of the cases, the osteoid osteoma has an atypical presentation (11). MRI is more sensitive in the diagnosis of bone marrow and soft tissue abnormalities adjacent to the lesion, but CT is more specific when it comes to detecting the lesion's nidus (3).

When osteoid osteoma is accompanied by severe inflammatory changes such as a prominent periosteal reaction, exaggerated synovial hypertrophy, and joint effusion, or extensive bone marrow and soft tissue edema, it may be difficult to reach a diagnosis. Findings such as these may cause radiologists to suspect an entity other than osteoid osteoma. For example, traumatic injury or infection may be suspected if small nidus is masked by extensive bone marrow and soft tissue edema (7).

Cortical thickening of the second, third and fourth metacarpal bones shown on the X-ray and MRI of our patient, in absence of clear visualization of the nidus, led the radiologist to describe it as a post-fracture callus. Indeed, when extensive cortical sclerosis is present in long bones, its appearance on radiographs can mimic both a stress fracture and osteoid osteoma. If the history and location of the cortical thickening are classic for a stress fracture, then additional workup is not indicated. However, if either the focal cortical thickening or the clinical presentation is atypical for a stress fracture, then further evaluation would be warranted, especially if there is no anamnestic data about the injury. In cases like this one, CT is excellent at demonstrating the presence of a subtle lucent stress fracture. The presence of a cortically 
based nidus, in addition to a characteristic clinical history, would indicate an osteoid osteoma, while the presence of a linear fracture lucency on CT would indicate a stress fracture (15). Bone scintigraphy as well may help differentiate a stress fracture from osteoid osteoma. On scintigraphic images, a stress fracture demonstrates linear, intense uptake of the tracer, whereas osteoid osteoma displays the "double-density" sign, in which intense central uptake is seen at the site of the nidus and moderate uptake is seen in the surrounding area (7).

Considering the possibility of spontaneous healing of osteoid osteoma, medicamental treatment in the form of prolonged use of nonsteroidal anti-inflammatory drugs is an option. However, caution is advised since there is a risk of the potential side effect of NSAID use. Furthermore, there are some reports which indicate that these tumors could progress to osteoblastoma on prolonged NSAID treatment. Complete surgical excision of the nidus, even if it is intralesional, is the treatment of choice for patients with severe pain, for those who do not respond to NSAIDs or aren't willing to tolerate pain, for patients with particular risk of long-term renal or gastrointestinal complications of NSAIDs, as well as for children when the constant presence of these tumors can lead to growth disorders. Surgical treatment provides immediate relief and is usually curative (9). Other less invasive methods of treatment have been described such as ablation of the nidus with a percutaneously placed radiofrequency, radionuclide-guided excision, CTguided percutaneous excision, percutaneous laser photocoagulation, and computer-assisted surgery (6).

\section{Conclusion}

Osteoid osteoma is a relatively rare cause of wrist pain but should be considered in young patients with persistent pain and untypical clinical or radiological findings. The radiologist must take anamnestic data and clinical findings into consideration when making a final conclusion on the diagnosis. If the nidus could not be clearly visualized by plain radiography, a CT scan should be obtained. We would like to emphasize the importance of communication between physicians and radiologists.

Authors' Contributions: Conception and design: $\mathrm{AH}$ and SJP; Acquisition, analysis and interpretation of data: SJP and JOM; Drafting the article: SM and AH; Revising it critically for important intellectual content: AH, SM, JOM and SJP; Approved final version of the manuscript: AH, SJP, JOM and SM.

Conflict of Interest: The authors declare that they have no conflict of interest.

\section{References}

1. Liu EH, Stone N, Alowami SO, Thoma A. Plast Reconstr Surg Glob Open. 2017;5(5):e1332.

2. Rolvien T, Zustin J, Mussawy H, Schmidt T, Pogoda P, Ueblacker P. Intra-articular osteoid osteoma as a differential diagnosis of diffuse monoarticular joint pain. BMC Musculoskelet Disord. 2016;17(1)

3. Hashemi J, Gharahdaghi M, Ansaripour E, Jedi F, Hashemi S. Radiological Features of Osteoid Osteoma: Pictorial Review. Iran J Radiol. 2011;08(03):182-89.

4. Dumitriu DI, Menten R, Clapuyt P. Pitfalls in the diagnosis of common benign bone tumours in children. Insights Imaging. 2014;5(6):645-55

5. Andalib A, and Sajadie-khajouei S. Osteoid osteoma of distal phalanx: A rare disorder and review of literature. J Res Med Sci. 2013; 18(3): 264-6.

6. Rajeev A, Ali M, Ralte A, Chakaravathy J. Osteoid osteoma as an unusual cause of wrist pain - A case report and review of literature. Int J Surg Case Rep. 2014;5(12):896-8.

7. Chai JW, Hong SH, Choi JY, Koh YH, Lee JW, Choi JA et al. Radiologic Diagnosis of Osteoid Osteoma: From Simple to Challenging Findings. Radiographics. 2010;30(3):737-49.

8. Gupta P, Rammohan R, Maini L, Gautam V, Khurana N. Osteoid Osteoma of the Meta- 
carpal-A Case Report. J Hand Microsurg. 2016;07(01):187-90.

9. Noordin S, Allana S, Hilal K, Nadeem N, Lakdawala R, Sadruddin A et al. Osteoid osteoma: Contemporary management. Orthop Rev. 2018;10(3).

10. Jafari D, Jamshidi K, Hajialilo Sami S, Shariatzadeh H, Najd Mazhar F, et al. Osteoid Osteoma of the Upper Extremity: a Report of 35 Cases, Shafa Ortho J. $2014 ; 1$ (1):e47.

11. Seniaray N, Jain A. Osteoid osteoma mimicking inflammatory synovitis. Indian J Nucl Med. 2017;32(3):194.
12. Kiers L, Shield L, Cole W. Neurological manifestations of osteoid osteoma.Arch Dis Child. 1990;65(8):851-855.

13. Rana R, Wu J, Eisenberg R. Periosteal Reaction. Am J Roentgenol. 2009;193(4):W259-W272.

14. Chronopoulos E, Xypnitos F, Nikolaou V, Efstathopoulos N, Korres D. Osteoid osteoma of a metacarpal bone: A case report and review of the literature. J Med Case Reports. 2008;2(1).

15. Shelat N, Shah R, Bennett DL. Cortically Based Sclerosis and Thickening. J Am Osteopath Coll Radiol. 2016;5(4):23-25. 